\title{
Virtual Spatial Channel Number and Index Modulation
}

\author{
Zahid Iqbal $\mathbb{D}^{1}{ }^{1}$ Fei Ji $\mathbb{D}^{1}{ }^{1}$ and Yun Liu $\mathbb{D}^{2}$ \\ ${ }^{1}$ School of Electronics and Information Engineering, South China University of Technology, Guangzhou 510640, China \\ ${ }^{2}$ School of Internet Finance and Information Engineering, Guangdong University of Finance, Guangzhou 510520, China
}

Correspondence should be addressed to Zahid Iqbal; zahidaryan777@gmail.com

Received 11 July 2021; Revised 13 August 2021; Accepted 18 August 2021; Published 13 September 2021

Academic Editor: Xiaojie Wang

Copyright (c) 2021 Zahid Iqbal et al. This is an open access article distributed under the Creative Commons Attribution License, which permits unrestricted use, distribution, and reproduction in any medium, provided the original work is properly cited.

This paper proposes a novel precoding-aided and efficient data transmission scheme called virtual spatial channel number and index modulation (VS-CNIM), which conveys extra data by changing both the number and index of active virtual parallel channels of multiple-input multiple-output (MIMO) channels, obtained through the singular value decomposition (SVD) in each time slot. Unlike the conventional virtual spatial modulation (VSM), where extra data bits are transmitted only using index of active virtual parallel channels, the VS-CNIM scheme, depending on incoming information bits, transmits extra bits utilizing both the number and indices of active parallel channels along the bits carried by $M$-ary constellation symbols. Therefore, VS-CNIM provides significantly superior spectral efficiency (SE) compared to VSM. Considering the influence of imperfect channel estimation, a closed-form upper bound is derived on average bit error probability (ABEP). The asymptotic performance is also analyzed, which gives the coding gain and diversity order and describes error floor under the consideration of perfect and imperfect channel estimation, respectively. Monte Carlo simulations exhibit that the VS-CNIM scheme achieves considerably better error performance and high SE than precoding-aided SM (PSM) and VSM schemes.

\section{Introduction}

Introduction of a multiple transmit and receive antennabased scheme in wireless communication systems referred to as multiple-input-multiple-output (MIMO) substantially improves the channel capacity [1-3]. Conventional MIMO schemes like vertical-Bell Laboratories layered space-time (V-BLAST) for multiplexing [4] and space-time block coding [5] for diversity gain have been studied to explore the advantages, whereas several problems inhibit the efficiency of MIMO systems, such as multiple radio frequency chain condition, interantenna synchronization, higher power requirement because of multiple antenna activation, interchannel interference (ICI), and complex decoding algorithms.

Spatial modulation (SM) [6-11] has been introduced to mitigate the above-mentioned problems inherent in MIMO systems and to achieve better data rates without sacrificing the end-to-end system performance of the communication system. SM uses a novel technique in which single transmit antenna activation is required for each transmission, thus minimizing the power requirements and reducing the trans- ceiver complexity making it beneficial for the practical applications $[12,13]$. The second feature that distinguishes SM from the MIMO schemes is the introduction of a novel coding method, which maps the spatial position of an active transmit antenna to the information block thus achieving a multiplexing gain over conventional SISO methods which also use a single antenna at a particular instant. A good number of methods have been studied following the basic idea of spatial modulation. In [14], space shift keying (SSK) is advanced, while reaping all the performance benefits of SM, it mitigates the implementation overhead of SM by using only the antenna index to relay information instead of transmitted symbols, which also minimizes the complexity in detection. To increase the spectral efficiencies of SM and SSK, generalized SM (GSM) [15] and generalized SSK (GSSK) [16] are proposed which allow the activation of multiple transmit antennae. Furthermore, in quadrature SM (QSM) [17], both real and imaginary subdomains are incorporated into a spatial domain, further increasing the spectral efficiency of SM. A low-complexity detection algorithm for index modulation multiple access (IM-MA) has 
been studied in [18]. In [19], a generalized QSM (GQSM) system to further increase the SE of QSM with a lowcomplexity detector as well as cooperative GQSM with OMA (C-OMA-GQSM) and cooperative GQSM with NOMA (C-NOMA-GQSM) schemes has been proposed. In [20-22], trellis-coded SM (TCSM) and space-time block coding SM (STBC-SM) schemes have been explored to increase the transmission diversity order of SM. In [23, 24], differential SM (DSM) is proposed to remove channel estimation at the receiver by conveying additional information using the antenna activation order.

In [25], precoding (preprocessing) SM (PSM) is designed to further extend the idea of SM to the receiver side. PSM employs zero-forcing processing or minimum mean squared error (MMSE) processing to obtain parallel channels. In PSM, a single receive antenna is activated for each time slot and extra information is conveyed implicitly via its index. PSM outperforms the conventional MIMO schemes as well as the transmitter side SM techniques at a minor cost of CSI requirement. Due to its outstanding performance among the conventional MIMO and SM schemes, an enormous research work has been proposed to further improve the performance of PSM [26-30]. As an extension to PSM, generalized PSM (GPSM) is proposed in [26], allowing multiple antennas to be activated in the PSM. Furthermore, in [27], Multistream Receive-Spatial Modulation (MSR-SM) which extends the GPSM scheme to multistream is introduced, achieving better performance in terms of coding gain and diversity as compared to state-of-the art MIMO schemes. Incorporating both in-phase and quadrature parts of the received signal, generalized precoding-aided quadrature SM (GPQSM) [28] can transmit additional information as compared with GPSM. The idea of PSM is applied in dual-hop hybrid SM (DH-HSM) [29] which uses the concept of spatial modulation in a dual-hop decode and forward protocol to convey information via multiple relays. Extending the work in [29], in [30], dual-hop hybrid DSM (DH-HDSM) is proposed which uses differential spatial modulation (DSM) in dual-hop networks with relaying to convey information. By avoiding channel estimation at all receiving nodes, it helps minimize the receiver complexity while maintaining the performance. In order to mitigate the limitations, such as elimination of ICI, number of transmit antennas, and high correlation between the parallel channels, of above-mentioned precoding schemes, a new precoding scheme called virtual spatial modulation (VSM) and its variants are proposed in [31-33] which exploits the "virtual parallel channels" to convey information. Virtual parallel channels are obtained by performing singular value decomposition on the MIMO channel. In the frequency domain, besides generalized multiple-mode orthogonal frequency division multiplexing with index modulation (GMMOFDM-IM) [34] and layered orthogonal frequency division multiplexing with index modulation (L-OFDM-IM) [35], a joint-mapping orthogonal frequency division multiplexing with subcarrier number modulation (JM-OFDM-SNM) has been recently proposed, which considers subcarrier activation patterns and constellation symbols jointly to improve the SE of the traditional OFDM system [36].
Inspired by the distinctive features of the VSM scheme, we propose a novel alternative scheme named virtual spatial channel number and index modulation (VS-CNIM), which through SVD converts the MIMO channels into less correlated virtual parallel channels and exploits not only the indices but also the number of virtual parallel channels hybridly to covey extra bits. For the purposes of precoding and detection, a right unitary matrix is utilized and a maximum likelihood (ML) detector is calculated, respectively. We examine the VS-CNIM scheme's average bit error probability (ABEP) while considering the influence of the imperfect channel estimation and Rayleigh fading assumption. More precisely, we calculate tight, closed-form expression for the upper bound on ABEP based on a union bounding method. Furthermore, motivated by $[37,38]$, in a high-SNR region, the asymptotic expression of ABEP is also derived, which in the presence of channel estimation errors describes the ABEP error floor and without channel estimation errors gives the coding gain and diversity order. Finally, the ABEP of the VS-CNIM scheme is investigated and compared with that of the state-of-the-art VSM scheme through Monte Carlo simulations, which show that the proposed scheme outperforms the VSM scheme in both performance and spectral efficiency (SE).

The rest of the paper is organized as follows. Section 2 introduces the general system model and overviews VSM. Section 3 explains the principle and transceiver structure design of the proposed VS-CNIM scheme. In Section 4, we derive the upper bound on ABEP as well as asymptotic analysis. Section 5 provides computer simulations and discussions, followed by conclusion in Section 6 .

1.1. Notations. Matrices are represented by boldface uppercase letters while column vectors are denoted by boldface lowercase letters. (. $)^{-1},(.)^{H}$, and $(.)^{T}$ are the inverse, Hermitian transpose, and transpose operations, respectively, while $\mathbb{C}$ is for the set of the complex number. $I_{K \times K},\|$.$\| , and$ $Q($.$) stand for the K \times K$ identity matrix, Frobenius norm, and $Q$-function, respectively. $P_{r}(),. \Gamma(),. f($.$) , and \lfloor$. represent the event's probability, gamma function, probability density, and floor functions, respectively. $\operatorname{sort}($.$) is$ the elements' reordering function, while rank (.) gives the matrix's rank and diag (.) produces the diagonal matrix from a vector.

This work was supported in parts by the National Natural Science Foundation of China under Grants 61431005 and 62071504 and by the Guangdong provincial research project under Grant 2016A030308006.

\section{General System Model and VSM Overview}

2.1. General System Model. In this paper, we consider a MIMO system with $N_{t}$ transmit and $N_{r}$ receive antennas employing a set $S$ of an $M$-ary constellation. The $H \in$ $\mathbb{C}^{N_{r} \times N_{t}}$ represents MIMO channels, where $h_{r, t}$ with $r \in\{1$, $\left.\cdots, N_{r}\right\}$ and $t \in\left\{1, \cdots, N_{t}\right\}$ represents the independent and identical channel gain element between the $r$-th receive and $t$-th transmit antennas, which is considered to be a zero mean and unit variance complex Gaussian random variable. 
In general, the precoded input-output received single vector can be written as

$$
\mathbf{y}=\mathbf{H P x}+\mathbf{z}
$$

where $\mathbf{y} \in \mathbb{C}^{N_{r} \times 1}$ is the receive single vector, $\mathbf{P} \in \mathbb{C}^{N_{t} \times N_{r}}$ is the precoding matrix, $\mathbf{x} \in \mathbb{C}^{N_{r} \times 1}$ is the transmit signal vector, and $\mathbf{z} \in \mathbb{C}^{N_{r} \times 1}$ is the additive white Gaussian noise having a covariance matrix $N_{0} I_{N_{r}}$ and mean zero.

2.2. VSM Overview. In this section, we overview the SM scheme called VSM, which was proposed to overcome the highly correlated nature of channels resulting from ZF or MMSE precoding in GPQSM. As VSM outperforms the precoding-aided GPQSM and hence is the state-of-the art scheme, hereafter we consider it a benchmark for discussion and comparison.

In VSM, out of $N_{r}$, only fixed $N_{l}$ virtual channels are activated for transmitting $M$-ary modulated data. In each time slot, a sum of $m=m_{1}+m_{2}$ information bits can be conveyed. Particularly, the $m_{1}=\left\lfloor\log _{2}\left(C\left(N_{r}, N_{l}\right)\right)\right\rfloor$ bits specify the $N_{l}$ active channel combination out of total $N_{r}$ channels, and the $m_{2}=N_{l} \log _{2}(M)$ are mapped to a symbol vector which is then transmitted over the selected active channels.

\section{Proposed VS-CNIM}

Following the general MIMO system model (1), we propose a new precoding scheme called VS-CNIM, to increase the spectral efficiency as well as error performance.

3.1. Principle and CSI of VS-CNIM. The widely famous parallel decomposition of the MIMO channel $\mathbf{H}$ is obtained by SVD as

$$
\mathbf{H}=\mathbf{U} \boldsymbol{\Lambda} \mathbf{V}^{H},
$$

where $\Lambda$ is the singular value matrix of dimension $N_{r} \times N_{t}$ and $\mathbf{U}$ and $\mathbf{V}$ are unitary matrices of sizes $N_{r} \times N_{r}$ and $N_{t} \times N_{t}$, respectively. Motivated by SVD, our purpose is to send extra spatial bits by exploiting the virtual parallel channels in $\boldsymbol{\Lambda}$. We can easily obtain the precoding matrix from $\mathbf{V}$, if at the receiver, there are perfect channel estimation and an ideal feedback of CSI to the transmitter from the receiver. Nonetheless, this supposition is not true in practical scenarios. Hence, for practical purposes, we consider limited feedback and imperfect channel estimation. The estimated matrix of the channel with errors is thus obtained by considering orthogonal pilots and ML to estimate the CSI and channel at the receiver, respectively. Finally, at the receiver, the erroneous estimated channel matrix can be modeled as $[39,40]$

$$
\overline{\mathbf{H}}=\mathbf{H}+\mathbf{H}_{*},
$$

where $\overline{\mathbf{H}}$ depends on $\mathbf{H}$, while $\mathbf{H}_{*}$ represents the channel error matrix and it does not depend on $\mathbf{H}$. The entries of $\mathbf{H}_{*}$ are i.i.d complex Gaussian random variables having mean zero and variance $\sigma_{*}^{2}$, while the correlation coefficient between $\overline{\mathbf{H}}$ and $\mathbf{H}$ is given by $\mathrm{Q}=1 /\left(1+\sigma_{*}^{2}\right)$. It is obvious that $\sigma_{*}^{2}=0$ is a unique case of perfect channel estimation. From the 1st-order autoregressive model, we obtain

$$
\mathbf{H}=\varrho \mathbf{H}+\mathbf{R},
$$

where $\mathbf{R}$ is a Gaussian random matrix with elements of zero mean and variance $(1-\varrho)$ and is independent of $\overline{\mathbf{H}}$. From CSI quantization [41], the limited feedback channel represented as $\tilde{\mathbf{H}}$ based on $\overline{\mathbf{H}}$ is given by

$$
\overline{\mathbf{H}}=\tilde{\mathbf{H}}+\mathbf{D}_{A} \text {, }
$$

where $\mathbf{D}_{A} \in \mathbb{C}^{N_{r} \times N_{t}}$ represents the noise matrix which is independent of $\tilde{\mathbf{H}}$ and having i.i.d random Gaussian variables as entries with zero mean and variance $\sigma_{A}^{2}$. Note that $\sigma_{A}^{2}$ denotes the average quantization distortion of the channel and $\sigma_{A}^{2}=0$ means a distortionless channel quantization. As $\mathbf{R}, \mathbf{D}_{A}$, and $\tilde{\mathbf{H}}$ are not dependent on each other, therefore by substituting (5) in (4), we get the true channel matrix via a limited feedback channel as

$$
\mathbf{H}=\mathrm{\varrho} \overline{\mathbf{H}}+\aleph,
$$

where $\aleph$ is of size $N_{r} \times N_{t}$ corresponding to the noise matrix possessing i.i.d complex Gaussian random variable elements of zero mean and variance $\varrho^{2} \sigma_{A}^{2}(1-\varrho)$. In (6), the channel estimation effect and the effect of the limited feedback channel are both contained into $\aleph$. Hence, for the sake of convenience, it is sensible to only regard the influence of imperfect channel estimation and ascribe the effect of the limited feedback channel to it $\left(=\tilde{\mathbf{H}}, \sigma_{A}^{2}\right)$. Based on the following different criteria, we chose the value of @: (1) fixed $\mathrm{Q}-$ for all SNRs, the estimation error is fixed, and (2) unfixed $\mathrm{Q}$-for any given SNR, the estimation error value changes by $\sigma_{*}^{2}=$ $1 /(p \eta)$, where $p$ represents the pilot symbol number utilized for channel estimation and $\eta=1 / N_{0}$ is the SNR [42]. The receiver has knowledge of $\overline{\mathbf{H}}$ regardless of $\mathbf{H}$ because of channel estimation; therefore, applying SVD on $\overline{\mathbf{H}}$, we get

$$
\overline{\mathbf{H}}=\overline{\mathbf{U}} \bar{\Lambda} \overline{\mathbf{V}}^{H}
$$

where $\bar{\Lambda}$ represents a $N_{r} \times N_{t}$ complex diagonal singular value matrix with diagonal elements arranged in descending order and $\overline{\mathbf{U}}$ and $\overline{\mathbf{V}}$ are, respectively, unitary matrices of dimensions $N_{r} \times N_{r}$ and $N_{t} \times N_{t}$. By the assumption of $N_{r} \leq N_{t}, \bar{\Lambda}$ has $N_{r}$ nonzero singular values; hence, (7) can be expressed as

$$
\mathbf{H}=\overline{\mathbf{U}}\left[\overline{\boldsymbol{\Lambda}}_{1}, 0\right]\left[\overline{\mathbf{V}}_{1}, \overline{\mathbf{V}}_{0}\right]^{H}=\overline{\mathbf{U}} \overline{\mathbf{\Lambda}}_{1} \overline{\mathbf{V}}_{1}^{H}
$$

where $\bar{\Lambda}_{1} \in \mathbb{C}^{N_{r} \times N_{r}}$ denotes a diagonal matrix with $N_{r}$ diagonal entries $\left\{\sqrt{\bar{\lambda}_{n}}\right\}_{n=1}^{N_{r}}$, showing virtual parallel channels; $\overline{\mathbf{V}}_{1} \in \mathbb{C}^{N_{t} \times N_{r}}$ and $\overline{\mathbf{V}}_{0} \in \mathbb{C}^{N_{t} \times\left(N_{t}-N_{r}\right)}$ comprise $N_{r}$ eigenvectors and $N_{t}-N_{r}$ singular vectors corresponding to $N_{r}$ nonzero and $N_{t}-N_{r}$ zero singular values, respectively. 


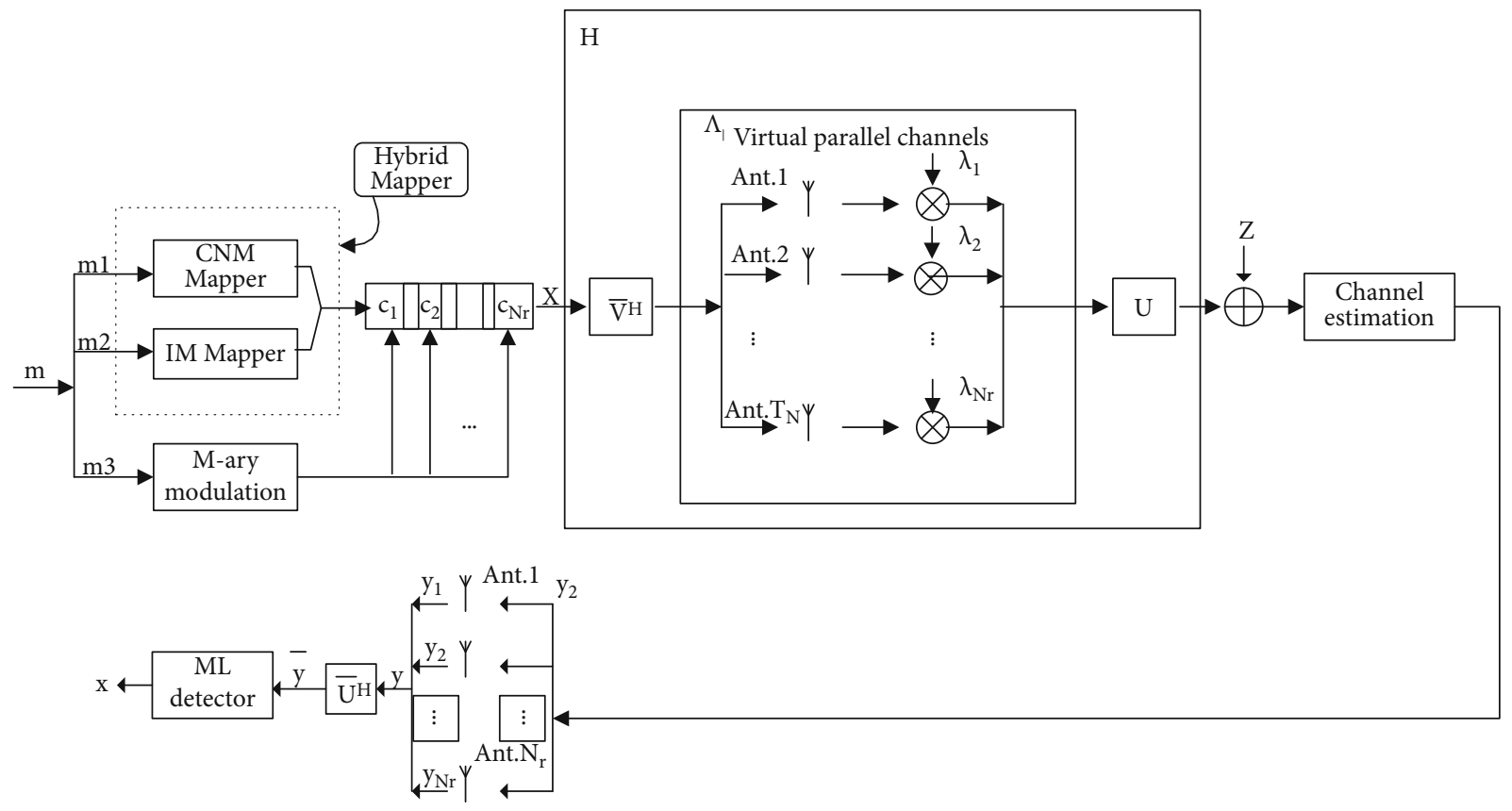

FIGURE 1: VS-CNIM transceiver structure.

Finally, we can obtain a receiver-side novel spatial modulation scheme by exploiting the $N_{r}$ virtual channels for transmitting extra spatial bits, feeding $\overline{\mathbf{V}}_{1}$ back to the transmitter for precoding and setting the signal vector as $\mathbf{x} \in \mathbb{C}^{N_{\mathrm{t}} \times 1}$.

3.2. VS-CNIM Transceiver. The transceiver structure of the proposed VS-CNIM is shown in Figure 1. During each time slot, a total number of $m=m_{1}+m_{2}+m_{3}$ data bits can be sent, where $m_{1}$ and $m_{2}$ are the nonconventional bits conveyed by the CNM and IM modulators, respectively. In each time slot, the CNM and IM mappers can be arranged arbitrarily because the hybrid mapper joins together both mappings regardless of their order. For suitable illustration of the basic VS-CNIM system model, we denote the CNM bits by $m_{1}$, which are used by the CNM mapper to determine the number of active parallel channels, and IM bits, denoted by $m_{2}$, are used to choose the indices of active channels by the IM mapper during each time slot. Then, using an appropriate mapping technique, the channel activation pattern (CAP) is determined through the combined CNM and IM mapping achieved by the hybrid mapper.

During each time slot, specific active channels are selected from the full set $\mathbb{N}=\left\{1,2, \cdots, N_{r}\right\}$ of $N_{r}$ virtual parallel channels based on the hybrid mapper $[43,44]$. The number of active channels at the $\tau$-th time slot $(N(\tau))$ is a variable that is dependent on the index and number.

The conventional $m_{3}=N(\tau) \log _{2}(M)$ bits are mapped to $M$-ary signal constellation for transmitting over $N(\tau)$ active channels. As an example, we provide a mapping table for mapping bits-to-CAP for $N_{r}=4$ with fixed length of $m_{1}=$ $m_{2}=\log _{2}\left(N_{r}\right)=2$ to simplify VS-CNIM system design in Table 1. The CAP at the $\tau$-th time slot can be expressed as $\mathbf{c}_{\tau}=\left[c(1) c(2) \cdots c\left(N_{r}\right)\right]^{T}$, where $c(j) \in\{0,1\}$ for $j=1,2, \cdots$, $N_{r}$. For instance, let us assume that at a certain time slot,
TABLE 1: Example of a lookup table of VS-CN-IM for $m_{1}=m_{2}=2$.

\begin{tabular}{lcccc}
\hline$\tau$ & $m_{1}$ & $m_{2}$ & $\mathbf{c}_{\tau}$ & $N(\tau)$ \\
\hline 1 & {$[00]$} & {$[00]$} & {$[1000]^{T}$} & 1 \\
2 & {$[00]$} & {$[01]$} & {$[0100]^{T}$} & 1 \\
3 & {$[00]$} & {$[10]$} & {$[0010]^{T}$} & 1 \\
4 & {$[00]$} & {$[11]$} & {$[0001]^{T}$} & 1 \\
5 & {$[01]$} & {$[00]$} & {$[1100]^{T}$} & 2 \\
6 & {$[01]$} & {$[01]$} & {$[1010]^{T}$} & 2 \\
7 & {$[01]$} & {$[10]$} & {$[1001]^{T}$} & 2 \\
8 & {$[01]$} & {$[11]$} & {$[0101]^{T}$} & 2 \\
9 & {$[10]$} & {$[00]$} & {$[1110]^{T}$} & 3 \\
10 & {$[10]$} & {$[01]$} & {$[1011]^{T}$} & 3 \\
11 & {$[10]$} & {$[10]$} & {$[1101]^{T}$} & 3 \\
12 & {$[10]$} & {$[11]$} & {$[0111]^{T}$} & 3 \\
13 & {$[11]$} & {$[00]$} & {$[0000]^{T}$} & 0 \\
14 & {$[11]$} & {$[01]$} & {$[0011]^{T}$} & 2 \\
15 & {$[11]$} & {$[10]$} & {$[0110]^{T}$} & 2 \\
16 & {$[11]$} & {$[11]$} & {$[1111]^{T}$} & 4 \\
\hline
\end{tabular}

the $m_{1}=[10]$ and $m_{2}=[01]$; then, $m_{1}$ gives the number of active channels which is 3 , and $m_{2}$ specifies the indices of these 3 active channels, i.e., $\left[\begin{array}{llll}1 & 0 & 1 & 1\end{array}\right]$.

Lastly, by considering the active channel combination and constellation symbol vector, a transmit signal vector is 
generated, which is then transmitted after precoding by $\mathbf{P}=\overline{\mathbf{V}}_{1}$.

At the receiver, multiplying the received signal vector by $\overline{\mathbf{U}}^{H}$, we get

$$
\overline{\mathbf{y}}=\overline{\mathbf{U}}^{H} \mathbf{y}=\overline{\mathbf{U}}^{H} \mathbf{H} \overline{\mathbf{V}}_{1} \mathbf{x}+\overline{\mathbf{U}}^{H} \mathbf{z} .
$$

Finally, by substituting (4) and (7) into (9) and doing some algebra, we obtain

$$
\overline{\mathbf{y}}=\mathrm{Q} \bar{\Lambda}_{1} \mathbf{x}+\overline{\mathbf{z}}
$$

where $\overline{\mathbf{z}}=\overline{\mathbf{U}}^{H} \mathbf{R} \overline{\mathbf{V}}_{1} \mathbf{x}+\overline{\mathbf{U}}^{H} \mathbf{z}$. As the probability distribution remains the same after multiplication of a matrix with a unitary matrix, therefore the probability distributions of $\overline{\mathbf{U}}^{H} \mathbf{R} \overline{\mathbf{V}}_{1} x$ and $\overline{\mathbf{U}}^{H} \mathbf{z}$ are the same as $\mathbf{R}$ and $\mathbf{z}$, respectively. Furthermore, as $\mathbf{R}$ and $\mathbf{z}$ are independent from each other, therefore each entry in $\overline{\mathbf{z}}$ has a complex Gaussian distribution of mean zero and variance $\varsigma=N_{0}+(1-\varrho)\|\mathbf{x}\|^{2}$.

Based on (10), two optimal detection methods are derived according to whether the correlation coefficient $\mathrm{Q}$ is known or not known to the receiver. When the receiver knows $\mathrm{Q}$, then the ML detector can be formulated as

$$
\mathbf{x} \wedge=\underset{\mathbf{x}}{\operatorname{argmin}}\left\|\overline{\mathbf{y}}-\mathrm{Q} \overline{\boldsymbol{\Lambda}}_{1} \mathbf{x}\right\|^{2} .
$$

Contrastingly, when the receiver does not know Q, then adequately a perfect channel estimation can be assumed; thus, by setting $\varrho=1$, the ML detector is obtained as

$$
\mathbf{x} \wedge=\underset{\mathbf{x}}{\operatorname{argmin}}\left\|\overline{\mathbf{y}}-\bar{\Lambda}_{1} \mathbf{x}\right\|^{2} .
$$

The near-ML detector proposed in [31] can be directly applied with a similar procedure to (11) and (12) in order to obtain low-complexity detection; therefore, it is ignored here. Moreover, the performance of (11) and (12) will be compared and analyzed in Section 5.

\section{Performance Analysis}

4.1. ABEP-Based Upper Bound Analysis. Considering the signal in (11), when $\mathbf{x}$ is transmitted on $\bar{\Lambda}$ and it is erroneously detected as $\mathbf{x}$, then the average PEP can be calculated as

$$
\begin{aligned}
\operatorname{Pr}\left(\mathbf{x} \longrightarrow \mathbf{x} \wedge \mid \bar{\Lambda}_{1}\right) & =\operatorname{Pr}\left(\left\|\overline{\mathbf{y}}-\mathrm{Q} \overline{\boldsymbol{\Lambda}}_{1} \mathbf{x}\right\|^{2}>\left\|\overline{\mathbf{y}}-\mathrm{Q} \overline{\boldsymbol{\Lambda}}_{1} \mathbf{x} \wedge\right\|^{2}\right) \\
& =Q\left(\sqrt{\frac{1}{2 \varsigma}\left\|\mathrm{Q} \overline{\boldsymbol{\Lambda}}_{1}(\mathbf{x}-\mathbf{x} \wedge)\right\|^{2}}\right) \\
& =Q\left(\sqrt{\frac{1}{2 \varsigma} \sum_{n=1}^{N_{r}} \bar{\lambda}_{n}\left|d_{n}\right|^{2}}\right),
\end{aligned}
$$

where $\lambda_{n}=\mathrm{Q}^{2} \bar{\sigma}_{n}^{2}, n \in\left\{1,2, \cdots, N_{r}\right\}$.
It is obvious from (13) that the joint PDF of $\bar{\Lambda}_{1}=$ $\left[\bar{\lambda}_{1}, \bar{\lambda}_{2}, \cdots, \bar{\lambda}_{N_{r}}\right]^{T}$ is required for the derivation of PEP, which is obtained by $[45,46]$ as

$$
\begin{aligned}
f\left(\bar{\Lambda}_{1}\right) & =\frac{1}{K} e^{-\sum_{n=1}^{N_{r}} \bar{\lambda}_{i}} \underbrace{\prod_{n=1}^{N_{r}} \bar{\lambda}_{n}^{N_{t}-N_{r}} \prod_{n+1}^{N_{r}}\left(\bar{\lambda}_{n}-\bar{\lambda}_{n+1}\right)^{2}}_{\mathrm{F}} \\
& =\frac{1}{K} \sum_{g=1}^{G} a_{g} e^{-\sum_{n=1}^{N_{r}} \bar{\lambda}_{n} \bar{\lambda}_{1}^{b_{g}^{1}} \cdots \bar{\lambda}_{N_{r}}^{N_{r}},}
\end{aligned}
$$

where $\quad K=\prod_{n=1}^{N_{r}}\left(\max \left\{N_{r}, N_{t}\right\}-n\right)\left(\min \left\{N_{r}, N_{t}\right\}-n\right)$ denotes the normalizing coefficient, the number of monomials in $\mathrm{F}$ is represented by $G, a_{g}$ with $g \in\{1,2, \cdots, G\}$ denotes the $g$-th monomial's coefficient in F, and $\left\{b_{g}^{n}\right\}_{n=1}^{N_{r}}$ represent the power coefficients of $\left\{\bar{\lambda}_{n}\right\}_{1}^{N_{r}}$ in the $g$-th monomial in F. Averaging (13) with (14) gives us

$$
\begin{aligned}
\operatorname{Pr}(x \longrightarrow x)= & E_{\bar{\Lambda}_{1}}\left[Q\left(\sqrt{\left.\frac{\sum_{n=1}^{N_{r} \bar{\lambda}_{n}\left|d_{n}\right|^{2}}}{2 \varsigma}\right)}\right]\right. \\
\cong & E_{\bar{\Lambda}_{1}}\left[\frac{1}{12} e^{-\sum_{n=1}^{N_{r}} \bar{\lambda}_{n}\left|d_{n}\right|^{2} / 4 \varsigma}+\frac{1}{4} e^{-\sum_{n=1}^{N_{r}} \bar{\lambda}_{n}\left|d_{n}\right|^{2} / 3 \varsigma}\right] \\
= & \frac{1}{12} \int e^{-\sum_{n=1}^{N_{r}} \bar{\lambda}_{n}\left|d_{n}\right|^{2} / 4 \varsigma} f\left(\bar{\Lambda}_{1}\right) d\left\{\bar{\Lambda}_{1}\right. \\
& +\frac{1}{4} \int e^{-\sum_{n=1}^{N_{r}} \bar{\lambda}_{n}\left|d_{n}\right|^{2} / 3 \varsigma} f\left(\bar{\Lambda}_{1}\right) d\left\{\bar{\Lambda}_{1},\right.
\end{aligned}
$$

where (15) is derived by the asymptotic identity $Q(x) \cong$ $(1 / 12) e^{-x^{2} / 2}+(1 / 4) e^{-2 x^{2} / 3}$ [47]. As per [48] (equation (8.312.2)), the first integral in (17) is given by

$$
\begin{aligned}
& \frac{1}{12} \int e^{-\sum_{n=1}^{N_{r}} \bar{\lambda}_{n}\left|d_{n}\right|^{2} / 4 \varsigma} f\left(\bar{\Lambda}_{1}\right) d\left\{\bar{\Lambda}_{1}\right. \\
& =\frac{1}{12 K} \sum_{g=1}^{G} a_{g} \int e^{-\sum_{n=1}^{N_{r}}\left(\left(\left|d_{n}\right|^{2} / 4 \varsigma\right)+1\right) \bar{\lambda}_{n}} \bar{\lambda}_{1}^{b_{g}^{1}} \ldots \bar{\lambda}_{N_{r}}^{N_{r}} d \bar{\Lambda}_{1} \\
& =\frac{1}{12 K} \sum_{g=1}^{G} a_{g} \int e^{-\left(\left(\left|d_{1}\right|^{2} / 4 \zeta\right)+1\right) \bar{\lambda}_{1}} \bar{\lambda}_{1}^{b_{g}^{1}} d \bar{\lambda}_{1} \\
& \quad \times \cdots \times \int e^{-\left(\left(\left|d_{N_{r}}\right|^{2} / 4 \zeta\right)+1\right) \bar{\lambda}_{N_{r}}} \bar{\lambda}_{N_{r}}^{N_{r}} d \bar{\lambda}_{N_{r}} \\
& =\frac{1}{12 K} \sum_{g=1}^{G} a_{g} \prod_{n=1}^{N_{r}}\left(\frac{\left|d_{n}\right|^{2}}{4 \varsigma}+1\right)^{-\left(b_{g}^{n}+1\right)} \Gamma\left(b_{g}^{n}+1\right) .
\end{aligned}
$$


Similarly, the second integral in (17) can be calculated as

$$
\begin{aligned}
& \frac{1}{4} \int e^{-\sum_{n=1}^{N_{r}} \bar{\lambda}_{n}\left|d_{n}\right|^{2} / 3 \varsigma} f\left(\bar{\Lambda}_{1}\right) d\left\{\bar{\Lambda}_{1}\right. \\
& \quad=\frac{1}{4 K} \sum_{g=1}^{G} a_{g} \prod_{n=1}^{N_{r}}\left(\frac{\left|d_{n}\right|^{2}}{3 \varsigma}+1\right)^{-\left(b_{g}^{n}+1\right)} \Gamma\left(b_{g}^{n}+1\right) .
\end{aligned}
$$

The unconditional PEP can be obtained by substituting (17) and (18) into (17) as follows:

$$
\begin{aligned}
\operatorname{Pr}(x \longrightarrow x) & \\
= & \frac{1}{4 K} \sum_{g=1}^{G} a_{g}\left(\prod_{n=1}^{N_{r}} \Gamma\left(b_{g}^{n}+1\right)\right) \\
& \times\left[\frac{1}{3} \prod_{n=1}^{N_{r}}\left(\frac{\left|d_{n}\right|^{2}}{4 \varsigma}+1\right)^{-\left(b_{g}^{n}+1\right)}+\prod_{n=1}^{N_{r}}\left(\frac{\left|d_{n}\right|^{2}}{3 \varsigma}+1\right)^{-\left(b_{g}^{n}+1\right)}\right] .
\end{aligned}
$$

Lastly, after getting the PEP, then by the famous union bounding technique of [49], an upper bound can be easily acquired as

$$
P_{e} \leq \frac{1}{m 2^{m}} \sum_{\mathbf{x}} \sum_{\mathbf{x} \wedge \neq \mathbf{x}} \operatorname{Pr}(\mathbf{x} \longrightarrow \mathbf{x} \wedge) N(\mathbf{x} \longrightarrow \mathbf{x} \wedge),
$$

where $N(\mathbf{x} \longrightarrow \mathbf{x} \wedge)$ denotes the number of error bits when $\mathbf{x}$ was transmitted and is erroneously detected as $\mathbf{x} \wedge$.

4.2. Analysis of Asymptotic ABEP. As noted earlier in Section 3.1, $\varsigma=N_{0}+(1-\varrho)\|\mathbf{x}\|^{2}$. Now, in the presence of channel estimation error, i.e., if $\varrho \neq 1$, then $\varsigma \longrightarrow \varsigma^{\star}=(1-\varrho)\|\mathbf{x}\|^{2}$, because $N_{0}=1 / \eta$ approaches zero as $\eta \longrightarrow+\infty$. According to $\varsigma=\varsigma^{*}$, the asymptotic ABEP can be derived by putting (18) into (19) as

$$
\begin{aligned}
P_{e} & \longrightarrow \frac{1}{m 2^{m+2} K} \sum_{\mathbf{x}} \sum_{\mathbf{x} \wedge \neq \mathbf{x}} N(\mathbf{x} \longrightarrow \mathbf{x} \wedge) \sum_{g=1}^{G} a_{g}\left(\prod_{n=1}^{N_{r}} \Gamma\left(b_{g}^{n}+1\right)\right) \\
\times & {\left[\frac{1}{3} \prod_{n=1}^{N_{r}}\left(\frac{\left|d_{n}\right|^{2}}{4 \varsigma^{\star}}+1\right)^{-\left(b_{g}^{n}+1\right)}+\prod_{n=1}^{N_{r}}\left(\frac{\left|d_{n}\right|^{2}}{3 \varsigma \star}+1\right)^{-\left(b_{g}^{n}+1\right)}\right] . }
\end{aligned}
$$

It can be observed from the above equation that in the region of high $\mathrm{SNR}$, the ABEP inclines to be uniform irrelevant to SNR, which in the ABEP performance gives an error floor.
Contrastingly, in the case of perfect channel estimation, where $\mathrm{Q}=1$, hence $\varsigma=N_{0}=1 / \eta$; thus, the asymptotic ABEP can be obtained as

$$
\begin{aligned}
P_{e} & \longrightarrow \frac{1}{m 2^{m+2} K} \sum_{\mathbf{x}} \sum_{\mathbf{x} \wedge \neq \mathbf{x}} N(\mathbf{x} \longrightarrow \mathbf{x} \wedge) \sum_{g=1}^{G} a_{g}\left(\prod_{n=1}^{N_{r}} \Gamma\left(b_{g}^{n}+1\right)\right) \\
& \times\left[\frac{1}{3} \prod_{n=1}^{N_{r}}\left(\frac{\left|d_{n}\right|^{2} \eta}{4}+1\right)^{-\left(b_{g}^{n}+1\right)}+\prod_{n=1}^{N_{r}}\left(\frac{\left|d_{n}\right|^{2} \eta}{3}+1\right)^{-\left(b_{g}^{n}+1\right)}\right] .
\end{aligned}
$$

It can be seen from (21) that similar to VSM, in VS-CNIM, the sum of terms having the largest exponent (similarly the smallest value among $\left\{b_{g}^{n}\right\}_{n=1}^{N_{r}}$ for all $g(s)$ of $\eta$ ) dominates the ABEP at high SNR, and when $r$ is minimum $(\min r=1)$, we get the worst case of PEP. Hence, the above equation can be further written as

$$
\begin{aligned}
P_{e} & \longrightarrow \frac{\eta^{-\mathcal{O}_{\min }}}{m 2^{m+2} K} \sum_{g, \mathscr{O}_{\min }} \sum_{s, s \wedge \epsilon \mathcal{S}} a_{g}\left(\prod_{n=1}^{N_{r}} \Gamma\left(b_{g}^{n}+1\right)\right) N(\mathbf{x} \longrightarrow \mathbf{x} \wedge) \\
& \times\left(\frac{|s-s \wedge|^{-2 \mathscr{O}_{\min }}}{3 \cdot 4^{-\mathscr{O}_{\min }}}+\frac{|s-s \wedge|^{-2 \mathscr{O}_{\min }}}{3^{-\mathcal{O}_{\min }}}\right)
\end{aligned}
$$

where $\sum_{g, \mathcal{O}_{\min }}$ represents the summation relating to all $g(s)$ for which $\mathcal{O}_{\text {min }}$ exists and $\mathcal{O}_{\min }=N_{t}-N_{r}+1$ indicates the diversity order. It can be seen from (22) that like in other precoding schemes, the configuration of MIMO antennas also determines the diversity order of VS-CNIM. Finally, the coding gain can be obtained from (22) by [50] as

$$
\begin{aligned}
C_{\text {gain }}= & {\left[\frac{1}{K m 2^{m+2}} \sum_{g, \mathscr{O}_{\min }} \sum_{s, s \wedge \in \mathcal{S}} a_{g}\left(\prod_{n=1}^{N_{r}} \Gamma\left(b_{g}^{n}+1\right)\right) N(\mathbf{x} \longrightarrow \mathbf{x} \wedge)\right.} \\
& \left.\times\left(\frac{|s-s \wedge|^{-2 \mathscr{O}_{\min }}}{3 \cdot 4^{-\mathscr{O}_{\min }}}+\frac{|s-s \wedge|^{-2 \mathscr{O}_{\min }}}{3^{-\mathscr{O}_{\min }}}\right)\right]^{-1 / \mathscr{O}_{\min }},
\end{aligned}
$$

which in the region of high SNR obeys

$$
P_{e} \cong\left(C_{\text {gain }} \eta\right)^{-\mathcal{O}_{\min }}
$$

\section{Simulation Results and Comparison}

In this section, we provide simulation results for the proposed VS-CNIM scheme with different system configurations and make the ABEP performance comparison with VSM [31]. We also investigate the performance of VSCNIM considering ideal and realistic channel conditions (equivalently, considering the effect of perfect and imperfect channel estimations).

The ABEP performance of the VS-CNIM scheme is compared with that of VSM in Figures 2 and 3, where BPSK 


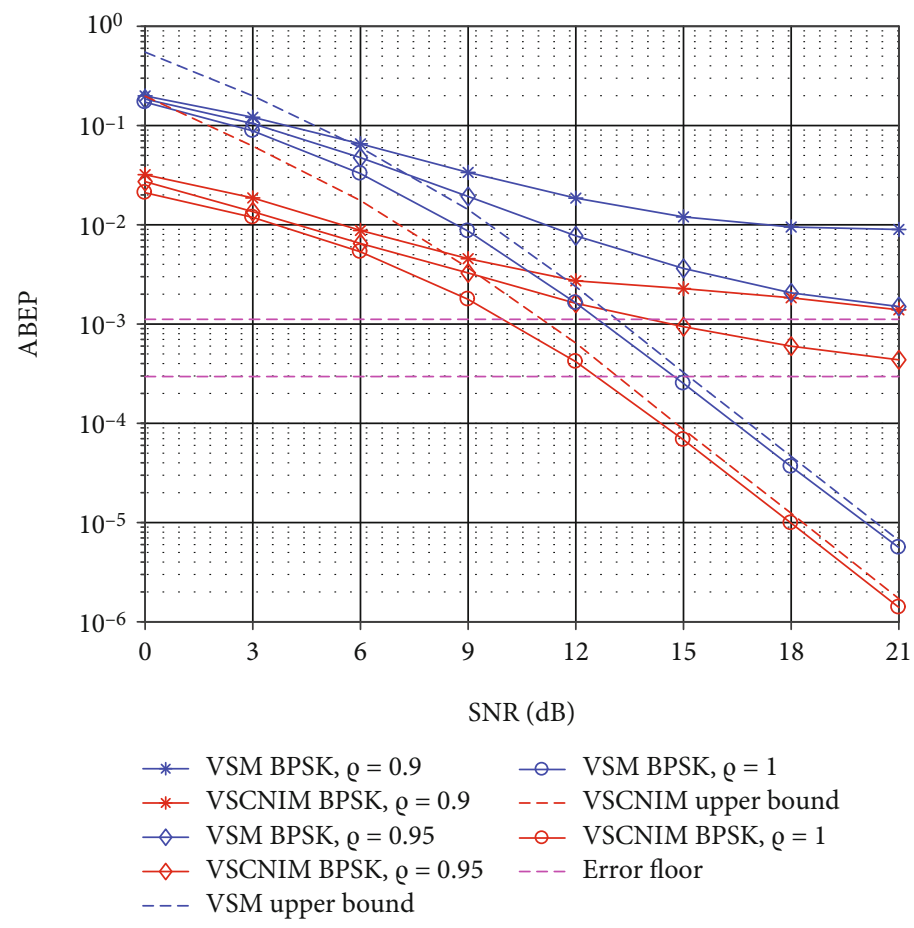

FIGURE 2: ABEP performance comparison between VS-CNIM and VSM at SE of 6.5 and 5 bits/s/Hz, respectively.

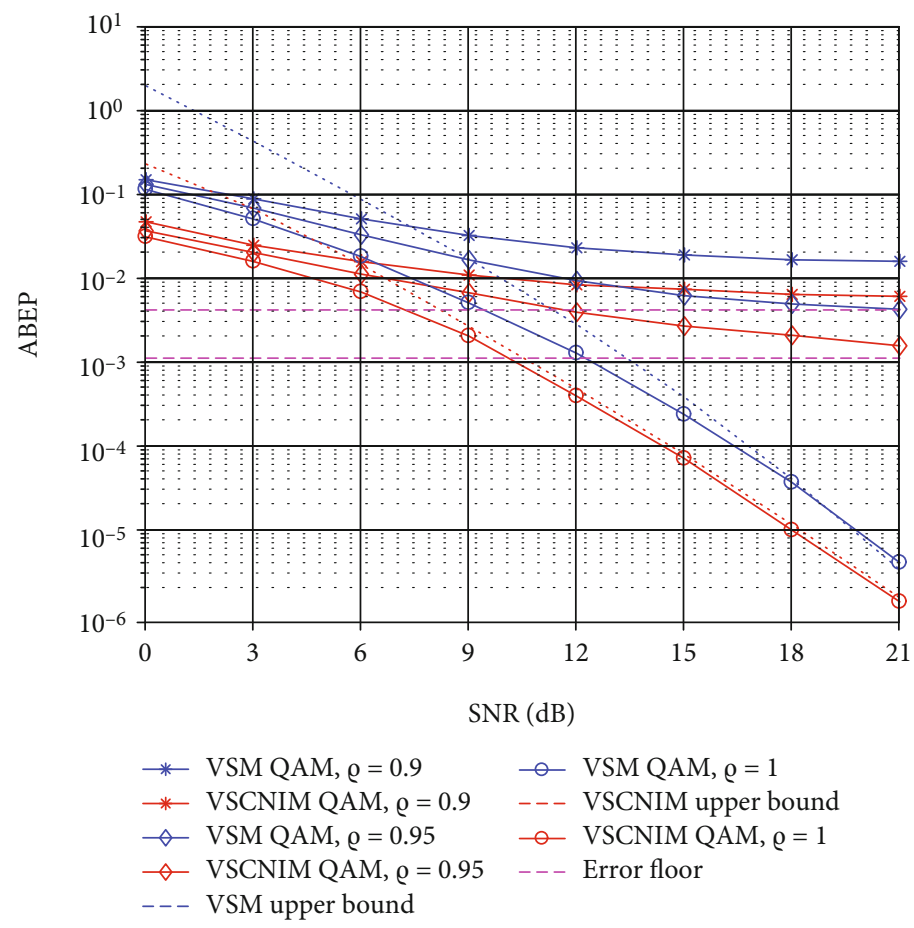

FIGURE 3: ABEP performance comparison between VS-CNIM and VSM at SE of 9 and $8 \mathrm{bits} / \mathrm{s} / \mathrm{Hz}$, respectively.

and 4QAM constellations are considered, respectively. The system parameters are set as $N_{t}=6$ and $N_{r}=4$ for both schemes, and 3 antennas are chosen as active for VSM. It is worth noting that the VS-CNIM achieves an average SE of $6.5 \mathrm{bits} / \mathrm{s} / \mathrm{Hz}$, and the SE of VSM is $5 \mathrm{bits} / \mathrm{s} / \mathrm{Hz}$ in
Figure 2, while in Figure 3, both schemes are compared at $\mathrm{SE}$ of $9 \mathrm{bits} / \mathrm{s} / \mathrm{Hz}$ and $8 \mathrm{bits} / \mathrm{s} / \mathrm{Hz}$, respectively. We consider $\mathrm{Q}=1,0.95$, and 0.9 in order to probe the effect of channel estimation, and the analytical ABEP upper bounds of VSCNIM for $\mathrm{Q}=1$ are also provided in both figures to verify 


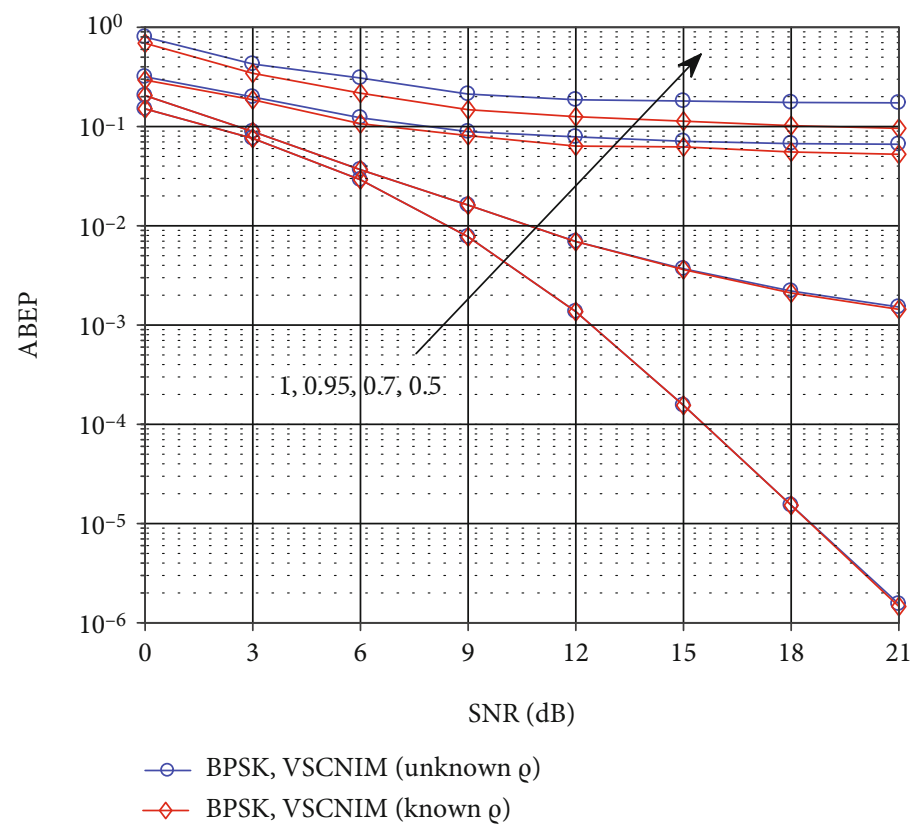

FIGURE 4: ABEP performance comparison of VS-CNIM receivers (11) and (12) with $\mathrm{Q}=1,0.95,0.7,0.5$.

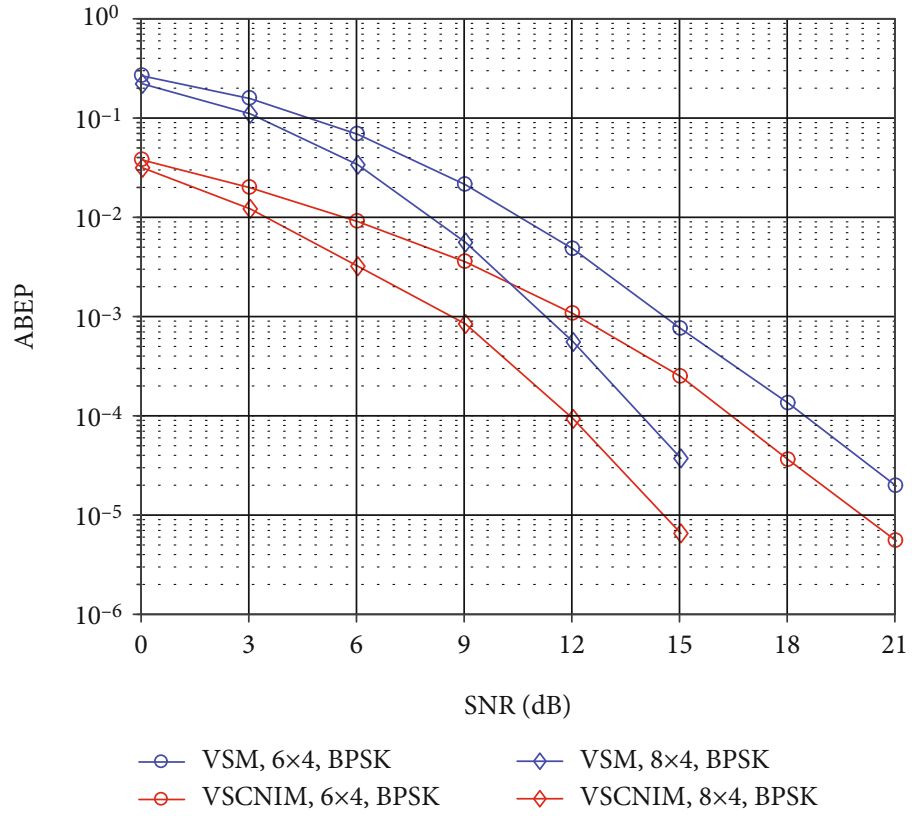

FIGURE 5: ABEP performance comparison between VS-CNIM and VSM with unfixed @ for two different system configurations: (a) $N_{t}=6$, $N_{r}=4$; (b) $N_{t}=8, N_{r}=4$.

our analysis, which become tighter as the SNR increases. The upper bounds for other values of $\mathrm{Q}$ are removed for figure clarity. Similar to VSM, VS-CNIM also gives error floors for $\mathrm{Q}=0.95$ and 0.9 , which are precisely reflected by analysis results. Furthermore, the error floor level for VS-CNIM is lower despite the fact that as the channel estimation error increases, the error floor also increases in both schemes. To justify this analysis, we recall (10), where we noted that instead of SNR, the channel estimation error scales the power of noise $\overline{\mathbf{z}}$. For all values of $\varrho$, VS-CNIM outperforms the counterpart in both error performance and SE at all SNRs, which can be acclaimed for transmitting extra index bits that are less likely to be corrupted.

In Figure 4, we compared the ABEP performance of the detector (11) and (12) of the VS-CNIM scheme in order to study the influence of known and unknown $\mathrm{Q}$ to the receiver with $N_{t}=6, N_{r}=4$, and fixed $\mathrm{Q}=1,0.95,0.7$, and 0.5 . It can be seen from the figure that when the values of $Q$ are large 
such as 1 and 0.95 , then irrespective of the knowledge of $\varrho$ to the detectors, the performance of both is almost the same. On the other hand, for smaller values of $\mathrm{Q}$ such as 0.7 and 0.5 , the detector having the knowledge of $\varrho$ outperforms the detector without having the knowledge of $\mathrm{Q}$. This can be explained by the fact that as @ gets smaller, then the impact of channel estimation gets critically notable and the knowledge of $\mathrm{Q}$ assists the receiver in precisely tracking the true channel. We can also observe that for smaller values of $\mathrm{Q}$, not only does the performance gap get visible but also the ABEP value becomes very large, which is unendurable in many communication applications. It is also often hard to obtain the true value of $\mathrm{Q}$; hence, receiver (12) is suggested for VS-CNIM which is simple to implement practically.

In Figure 5, the ABEP performance of VS-CNIM is examined and compared with that of VSM under another channel estimation model for two system configurations with unfixed $\mathrm{Q}$, which as per $\rho=p \eta /(p \eta+1)$ changes with SNR. The system configurations are (a) $N_{t}=6$ and $N_{r}=4$ and (b) $N_{t}=8$ and $N_{r}=4$ and the pilot symbol number $p=2$. It can be seen from the figure that for both VSCNIM and VSM schemes, the error floors nevermore occur when $\varrho$ is unfixed which is unlike the previous study of scenario where $\mathrm{Q}$ was fixed. The reason for it is that in the region of high SNR, @ tends to be 1, which is the case of perfect channel estimation. However, the figure depicts that similar to the case of fixed $\mathrm{Q}$, VS-CNIM also outperforms VSM for all SNRs in the case of unfixed $\mathrm{Q}$.

\section{Conclusions and Future Work}

In this paper, a novel better error performance and spectral efficient SM scheme, namely, VS-CNIM, has been proposed, which conveys extra spatial bits by utilizing both the number and indices of active parallel channels obtained from the decomposition of MIMO channels. For the performance evaluation of VS-CNIM, we have calculated a closed-form upper bound on the ABEP. Moreover, under the consideration of perfect and imperfect channel estimation, the ABEPs have been also examined, based on which the diversity order and the resulted error floor of the proposed scheme are described. Lastly, simulation results have depicted that the proposed scheme outperforms the VSM scheme significantly in both SE and error performance.

In future work, a transmitter-side VS-CNIM scheme with $N r>N_{t}$ will be researched, where the same performance analysis is also valid. In the transmitter-side VSCNIM scheme, instead of $N r$, the $N_{t}$ virtual parallel channels are utilized for the purpose of channel number and index modulation.

\section{Data Availability}

The mathematical derivations and result discussions used to support the findings of this study are included within the article along necessary citations, while the MATLAB code used to obtain the simulation results of this study is available from the corresponding author upon request.

\section{Conflicts of Interest}

The authors declare that they have no conflicts of interest.

\section{References}

[1] E. Telatar, "Capacity of multi-antenna Gaussian channels," European Transactions on Telecommunications, vol. 10, no. 6, pp. 585-595, 1999.

[2] D. Tse and P. Viswanath, Fundamentals of Wireless Communication, Cambridge university press, 2005.

[3] X. Cheng, B. Yu, L. Yang et al., "Communicating in the real world: 3D MIMO," IEEE Wireless Communications, vol. 21, no. 4, pp. 136-144, 2014.

[4] P. W. Wolniansky, G. J. Foschini, G. D. Golden, and R. A. Valenzuela, "V-blast: an architecture for realizing very high data rates over the rich-scattering wireless channel," in 1998 URSI international symposium on signals, systems, and electronics. Conference proceedings (Cat. No. 98EX167), pp. 295300, Pisa, Italy, 1998.

[5] V. Tarokh, H. Jafarkhani, and A. Robert Calderbank, "Spacetime block coding for wireless communications: performance results," IEEE Journal on Selected Areas in Communications, vol. 17, no. 3, pp. 451-460, 1999.

[6] M. Di Renzo, H. Haas, and P. M. Grant, "Spatial modulation for multiple-antenna wireless systems: a survey," IEEE Communications Magazine, vol. 49, no. 12, pp. 182-191, 2011.

[7] P. Yang, M. Di Renzo, Y. Xiao, S. Li, and L. Hanzo, "Design guidelines for spatial modulation," IEEE Communications Surveys \& Tutorials, vol. 17, no. 1, pp. 6-26, 2014.

[8] P. Yang, Y. Xiao, Y. L. Guan et al., "Single-carrier SM-MIMO: a promising design for broadband large-scale antenna systems," IEEE Communications Surveys \& Tutorials, vol. 18, no. 3, pp. 1687-1716, 2016.

[9] M. Di Renzo, H. Haas, A. Ghrayeb, S. Sugiura, and L. Hanzo, "Spatial modulation for generalized MIMO: challenges, opportunities, and implementation," Proceedings of the IEEE, vol. 102, no. 1, pp. 56-103, 2013.

[10] E. Basar, M. Wen, R. Mesleh, M. di Renzo, Y. Xiao, and H. Haas, "Index modulation techniques for next-generation wireless networks," IEEE access, vol. 5, pp. 16693-16746, 2017.

[11] M. Wen, B. Zheng, K. J. Kim et al., "A survey on spatial modulation in emerging wireless systems: research progresses and applications," IEEE Journal on Selected Areas in Communications, vol. 37, no. 9, pp. 1949-1972, 2019.

[12] A. Younis, W. Thompson, M. Di Renzo et al., "Performance of spatial modulation using measured real-world channels," in 2013 IEEE 78th vehicular technology conference (VTC fall), pp. 1-5, Las Vegas, NV, USA, 2013.

[13] N. Serafimovski, A. Younis, R. Mesleh et al., "Practical implementation of spatial modulation," IEEE Transactions on Vehicular Technology, vol. 62, no. 9, pp. 4511-4523, 2013.

[14] J. Jeganathan, A. Ghrayeb, L. Szczecinski, and A. Ceron, "Space shift keying modulation for MIMO channels," IEEE Transactions on Wireless Communications, vol. 8, no. 7, pp. 3692-3703, 2009.

[15] J. Jeganathan, A. Ghrayeb, and L. Szczecinski, "Generalized space shift keying modulation for MIMO channels," in 2008 IEEE 19th International Symposium on Personal, Indoor and Mobile Radio Communications, pp. 1-5, Cannes, France, 2008.

[16] A. Younis, N. Serafimovski, R. Mesleh, and H. Haas, "Generalised spatial modulation," in 2010 conference record of the forty 
fourth Asilomar conference on signals, systems and computers, pp. 1498-1502, Pacific Grove, CA, USA, 2010.

[17] R. Mesleh, S. S. Ikki, and H. M. Aggoune, "Quadrature spatial modulation," IEEE Transactions on Vehicular Technology, vol. 64, no. 6, pp. 2738-2742, 2014.

[18] J. Li, Q. Li, S. Dang, M. Wen, X. Q. Jiang, and Y. Peng, "Lowcomplexity detection for index modulation multiple access," IEEE Wireless Communications Letters, vol. 9, no. 7, pp. 943947, 2020.

[19] J. Li, S. Dang, Y. Yan, Y. Peng, S. Al-Rubaye, and A. Tsourdos, "Generalized quadrature spatial modulation and its application to vehicular networks with NOMA," IEEE Transactions on Intelligent Transportation Systems, vol. 22, no. 7, pp. 40304039, 2020.

[20] R. Mesleh, M. Di Renzo, H. Haas, and P. M. Grant, "Trellis coded spatial modulation," IEEE Transactions on Wireless Communications, vol. 9, no. 7, pp. 2349-2361, 2010.

[21] E. Basar, U. Aygolu, E. Panayirci, and H. Vincent Poor, "New trellis code design for spatial modulation," IEEE Transactions on Wireless Communications, vol. 10, no. 8, pp. 2670-2680, 2011.

[22] E. Başar, U. Aygölü, E. Panayirci, and H. V. Poor, "Space-time block coded spatial modulation," IEEE Transactions on Communications, vol. 59, no. 3, pp. 823-832, 2010.

[23] Y. Bian, X. Cheng, M. Wen, L. Yang, H. V. Poor, and B. Jiao, "Differential spatial modulation," IEEE Transactions on Vehicular Technology, vol. 64, no. 7, pp. 3262-3268, 2014.

[24] J. Li, M. Wen, X. Cheng, Y. Yan, S. Song, and M. H. Lee, "Differential spatial modulation with gray coded antenna activation order," IEEE Communications Letters, vol. 20, no. 6, pp. 1100-1103, 2016.

[25] L.-L. Yang, "Transmitter preprocessing aided spatial modulation for multiple-input multiple-output systems," in 2011 IEEE 73rd vehicular technology conference (VTC spring), pp. 1-5, Budapest, Hungary, 2011.

[26] R. Zhang, L.-L. Yang, and L. Hanzo, "Generalised pre-coding aided spatial modulation," IEEE Transactions on Wireless Communications, vol. 12, no. 11, pp. 5434-5443, 2013.

[27] A. Stavridis, M. Di Renzo, and H. Haas, "Performance analysis of multistream receive spatial modulation in the MIMO broadcast channel," IEEE Transactions on Wireless Communications, vol. 15, no. 3, pp. 1808-1820, 2015.

[28] L. Xiao, P. Yang, S. Fan, S. Li, L. Song, and Y. Xiao, "Lowcomplexity signal detection for large-scale quadrature spatial modulation systems," IEEE Communications Letters, vol. 20, no. 11, pp. 2173-2176, 2016.

[29] A. Stavridis, D. Basnayaka, S. Sinanovic, M. Di Renzo, and H. Haas, "A virtual MIMO dual-hop architecture based on hybrid spatial modulation," IEEE Transactions on Communications, vol. 62, no. 9, pp. 3161-3179, 2014.

[30] M. Zhang, M. Wen, X. Cheng, and L. Yang, "A dual-hop virtual MIMO architecture based on hybrid differential spatial modulation," IEEE Transactions on Wireless Communications, vol. 15, no. 9, pp. 6356-6370, 2016.

[31] J. Li, M. Wen, M. Zhang, and X. Cheng, "Virtual spatial modulation,” IEEE Access, vol. 4, pp. 6929-6938, 2016.

[32] Q. Li, M. Wen, J. Li, X. Cheng, and F. Chen, "Virtual spatial modulation with diversity improvement," in 2017 IEEE 86th vehicular technology conference (VTC-fall), pp. 1-5, Toronto, ON, Canada, 2017.

[33] X. Gong, M. Wen, Z. Iqbal, J. Li, S. Mumtaz, and A. AlDulaimi, "Multiple-mode MIMO with index modulation and its inphase/quadrature extension," in ICC 2020-2020 IEEE International Conference on Communications (ICC), pp. 1-6, Dublin, Ireland, 2020.

[34] M. Wen, Q. Li, E. Basar, and W. Zhang, "Generalized multiplemode OFDM with index modulation," IEEE Transactions on Wireless Communications, vol. 17, no. 10, pp. 6531-6543, 2018.

[35] J. Li, S. Dang, M. Wen, X.-Q. Jiang, Y. Peng, and H. Hai, "Layered orthogonal frequency division multiplexing with index modulation," IEEE Systems Journal, vol. 13, no. 4, pp. 3793-3802, 2019.

[36] M. Wen, J. Li, S. Dang, Q. Li, S. Mumtaz, and H. Arslan, “Jointmapping orthogonal frequency division multiplexing with subcarrier number modulation," IEEE Transactions on Communications, vol. 69, no. 7, pp. 4306-4318, 2021.

[37] M. Di Renzo and H. Haas, "Bit error probability of SM-MIMO over generalized fading channels," IEEE Transactions on Vehicular Technology, vol. 61, no. 3, pp. 1124-1144, 2012.

[38] M. Di Renzo and H. Haas, "On transmit diversity for spatial modulation MIMO: impact of spatial constellation diagram and shaping filters at the transmitter," IEEE Transactions on Vehicular Technology, vol. 62, no. 6, pp. 2507-2531, 2013.

[39] D. Samardzija and N. Mandayam, "Pilot-assisted estimation of MIMO fading channel response and achievable data rates," IEEE Transactions on Signal Processing, vol. 51, no. 11, pp. 2882-2890, 2003.

[40] B. Hassibi and B. M. Hochwald, "How much training is needed in multiple-antenna wireless links?," IEEE Transactions on Information Theory, vol. 49, no. 4, pp. 951-963, 2003.

[41] D. Zhang, W. Guo, J. Zhu, and Z. Tian, "On the bounds of feedback rates for pilot-assisted MIMO systems," IEEE Transactions on Vehicular Technology, vol. 56, no. 4, pp. 1727-1736, 2007.

[42] W. M. Gifford, M. Z. Win, and M. Chiani, "Diversity with practical channel estimation," IEEE Transactions on Wireless Communications, vol. 4, no. 4, pp. 1935-1947, 2005.

[43] A. M. Jaradat, J. M. Hamamreh, and H. Arslan, "Modulation options for OFDM-based waveforms: classification, comparison, and future directions," IEEE Access, vol. 7, pp. 1726317278, 2019.

[44] A. M. Jaradat, J. M. Hamamreh, and H. Arslan, "OFDM with hybrid number and index modulation," IEEE Access, vol. 8, pp. 55042-55053, 2020.

[45] A. T. James, "Distributions of matrix variates and latent roots derived from normal samples," The Annals of Mathematical Statistics, vol. 35, no. 2, pp. 475-501, 1964.

[46] L. G. Ordonez, D. P. Palomar, A. Pages-Zamora, and J. Fonollosa, "High-SNR analytical performance of spatial multiplexing MIMO systems with CSI," IEEE Transactions on Signal Processing, vol. 55, no. 11, pp. 5447-5463, 2007.

[47] M. Chiani and D. Dardari, "Improved exponential bounds and approximation for the q-function with application to average error probability computation," in Global Telecommunications Conference, 2002. GLOBECOM'02. IEEE, vol. 2, pp. 13991402, Taipei, Taiwan, 2002.

[48] D. Zwillinger and A. Jeffrey, Table of Integrals, Series, and Products, Elsevier, 2007.

[49] J. G. Proakis, Digital Communications, Mc Graw-Hill, 2001.

[50] Z. Wang and G. B. Giannakis, "A simple and general parameterization quantifying performance in fading channels," IEEE Transactions on Communications, vol. 51, no. 8, pp. 13891398, 2003. 